\title{
Microbiological analysis and determination of antibacterial activity of apple samples collected from local markets in Dhaka city, Bangladesh
}

\author{
Sheikh Al Mamun, Kamal Kanta Das and Md. Aftab Uddin* \\ Department of Microbiology, Stamford University Bangladesh, 51 Siddeswari Road, Dhaka 1217, Bangladesh
}

Received 02 April 2016/Accepted 29 April 2016

\begin{abstract}
Present study attempted to detect the prevalence of contaminating microorganisms in 10 categories of local market apple samples commonly consumed in Dhaka city, Bangladesh. A significant number of total viable bacteria were quantified up to $10^{6} \mathrm{cfu} / \mathrm{g}$ in these samples. Staphylococcus spp. was the predominant isolate within a range of $1.0 \times 10^{4}-3.2 \times 10^{6} \mathrm{cfu} / \mathrm{g}$ whereas Escherichia coli, Klebsiella spp., Salmonella spp., Shigella spp., Pseudomonas spp. and Vibrio spp. were also quantified. Fungal contamination (within the range of $10^{5} \mathrm{cfu} / \mathrm{g}-10^{7} \mathrm{cfu} / \mathrm{g}$ ) was also observed. Alarmingly most of the bacterial isolates present in the samples were proved to be multi-drug resistant. Antibacterial activity of the samples was tested against some common bacterial isolates but was found to be ineffective.
\end{abstract}

Key words: Apple; Contamination; Drug resistance; Antimicrobial activity

Apples are a well-off source of different sorts of phytochemicals including flavonoids (e.g., catechins, flavanols etc) with other phenolic compounds like epicatechin- procyanidins available in the skin, core and pulp of different types of apple. Current research is trying to investigate whether nutrients and/or phytochemicals in apples may impinge on the risk of some types of cancers. Apples become readily contaminated at different stages during its production such as growing, harvesting, processing, storing, shipping or preparing (1-3). Presence of bacterial isolates possess a potential threat of food borne illness to consumers. Pathogens such as Escherichia coli, Salmonella spp., Listeria monocytogenes, Aeromonas spp., Staphylococcus spp., Streptococcus spp., Vibrio spp. and Pseudomonas are responsible for various disease, morbidity and mortality. Salmonella spp. alone is responsible for 1.3 billion cases of salmonellosis and 3 million deaths $(4,5)$.

One of the most important health related concern on fruit consumption lies on the occurrence of drug resistant microorganisms which is known to broadly hamper the infectious disease medication that is mainly based on the use of antibiotics (6-11). In recent years, many antibiotics have lost their efficiency due to development of resistant strains (12) and through the resistance genes expression and also due to natural mutations within the microbial populations (13).

${ }^{*}$ Corresponding Author: Mailing address. Md. Aftab Uddin, Department of Microbiology, Stamford University Bangladesh, 51 Siddeswari Road, Dhaka 1217, Bangladesh, Bangladesh; E-mail: aftab_mb12@yahoo.com.
This situation has lead to the development of many adverse effects of antibiotics including hypersensitivity, immune-suppression and allergic reactions (14) and increasing interest in mounting alternative antimicrobial drugs which are highly effective and non toxic, derived entirely from natural sources (15). Ultimately, scientists are gaining interest towards natural products that have been the scientific basis of remedial effects (16).

According to the World Health Organization, about $80 \%$ of the world's population still depends entirely on plants for their medication. In both developing and developed nations medicinal plants are the main source of drugs. Beside plants, fruits also have been studied by the researchers for the detection of many active phytochemicals such as polyhenols, anthocyanins, flavonoids, terpenoids, carotenoids, cumarins, saponin and vitamins that are richly present in fruits (17).

Based on these facts and considerations, present study aimed to determine and enumerate microorganisms causing the spoilage of apples with their drug-resistance traits and to assess the anti-bacterial activity of the samples studied.

\section{MATERIALS AND METHODS}

Selection of study area \& collection of fruit samples for investigation.
Samples were collected from some popular super shops from five areas of Dhaka
city such as Rampura, Khilgao, Shantinagar, Moghbazar and Mailbagh. Samples
were collected in different time intervals and transported to the laboratory as soon
as possible according to the method suggested by American Public Health
Association (18). For the identification and enumeration of pathogenic bacteria
and fungi, at first $10 \mathrm{~g}$ of each sample was taken, then blended with $90 \mathrm{ml}$ normal
saline (pH 7.8) and diluted up to $10^{-4}$ and then dilutions were used for plating
purposes according to the standard guideline (19).
Isolation and enumeration of spoiling microorganisms. The primary
inoculation was performed by the conventional culture technique with the addition 
of $0.1 \mathrm{ml}$ of each sample onto nutrient agar (NA) and Sabouraud's Dextrose Agar (SDA) for the determination total viable bacterial count (TVBC) and total fungal load respectively, following the spread plate technique (19). The plates were incubated at $37{ }^{\circ} \mathrm{C}$ for 24 hours and at $25{ }^{\circ} \mathrm{C}$ for 48 hours for TVBC and fungal load respectively. From the dilutions $10^{-3}$ and $10^{-5}$, the sample $(0.1 \mathrm{ml})$ was spread onto MacConkey agar for the enumeration of coliforms (especially, Escherichia coli and Klebsiella spp.) and fecal coliform, respectively. Afterward, the MacConkey agar plates were incubated at $37{ }^{\circ} \mathrm{C}$ for 24 hours. An aliquot of $0.1 \mathrm{ml}$ of diluted sample was spread onto Mannitol Salt Agar (MSA) and Pseudomonas agar for the isolation of Staphylococcus spp. And Pseudomonas spp. respectively and incubated at $37{ }^{\circ} \mathrm{C}$ for 24 hours. For the enumeration of Listeria spp., $0.1 \mathrm{ml}$ of suspension was spread onto the Listeria identification media and plates were incubated at $37{ }^{\circ} \mathrm{C}$ for 24 hours. Listeria spp. was identified as blue-green colonies on Listeria identification agar media with a further confirmation by biochemical tests (20).

Enrichment for enumeration of Salmonella spp., Shigella spp. and Vibrio spp. Prior to quantify the relatively stressed cells or the viable but nonculturable (VBNC) microbial cells, $1 \mathrm{ml}$ of sample was transferred into $9 \mathrm{ml}$ of selenite cysteine broth (SCB) and alkaline peptone water (APW) for the enrichment of Salmonella, Shigella, and Vibrio spp. respectively and incubated at $37{ }^{\circ} \mathrm{C}$ for 6 hours $(8,21,22)$. After incubation, the samples were diluted up to $10^{-5}$ and then $0.1 \mathrm{ml}$ of samples from $10^{-3}$ and $10^{-5}$ dilutions were spread onto Salmonella-Shigella (SS) agar and Thiosulfate Citrate Bile Salt Sucrose (TCBS) agar for the isolation of Salmonella and Shigella spp, and Vibrio spp. respectively. Plates were incubated at $37{ }^{\circ} \mathrm{C}$ for 48 hours for the detection of typical colonies. Finally, all the isolates were biochemically examined following standard procedures $(8,19,23)$

Antibiotic susceptibility test. All the bacterial isolates were examined for antibiotic susceptibility traits (either drug resistant or sensitive) against 16 antibacterial drugs (including first, second and third generation drugs) by disc diffusion assay on Mueller-Hinton agar (Difco, Detroit, MI) agains commonly used antibiotics following the standard protocol $(24,25)$. Antibiotic discs included trimethoprime/sulfamethoxazole $(25 \mu \mathrm{g})$, erythromycin $(15 \mu \mathrm{g})$, amoxicillin $(30 \mu \mathrm{g})$, ceftriaxone $(30 \mu \mathrm{g})$, ciprofloxacin $(5 \mu \mathrm{g})$, streptomycin $(10 \mu \mathrm{g})$, ampicillin $(10 \mu \mathrm{g})$, tetracycline $(30 \mu \mathrm{g})$, chloramphenicol $(30 \mu \mathrm{g})$, cefixime $(5 \mu \mathrm{g})$, polymixin b (300 units), kanamycine $(30 \mu \mathrm{g})$, vancomycine $(30 \mu \mathrm{g})$, gentamicin $(10 \mu \mathrm{g})$, nalidixic acid $(30 \mu \mathrm{g})$, azythromycine $(15 \mu \mathrm{g})$ and penicillin $\mathrm{G}(10 \mu \mathrm{g})$.

Determination of antibacterial activity of apple samples. Antibacteria activity was determined by using the agar well diffusion methods. (26, 27) Normal saline suspensions of test organisms (Pseudomonas spp., Listeria spp., Aeromonas spp., Vibrio spp., Salmonella spp., Klebsiella spp., Staphylococcus aureus, E. coli) consisting of $10^{6}$ cells $/ \mathrm{mL}$ (compared with McFarland standard) were introduced on separate Muller- Hinton agar (MHA) and lawns were prepared. After drying, sterile cork borers were used to create $8 \mathrm{~mm}^{3}$ wells. A volume of $100 \mu \mathrm{L}$ of each sample (with a concentration of $11 \mu \mathrm{g} / \mu \mathrm{L}$ ) was poured into separate wells, dried and then incubated at $37{ }^{\circ} \mathrm{C}$ for 12-18 hours. Normal saline and Chloramphenicol $(10 \mu \mathrm{g})$ were used as positive and negative control, respectively. Presence of clear zone around the sample solution (if any) indicated the presence of antibacterial activity.

\section{RESULTS AND DISCUSSIONS}

Fruits are mostly eaten raw. So they may lead to the outbreaks of human diseases causing the overall public health at a serious threat. Many health-related problems are associated with the propagation of etiological agents as well as their drug-resistance abilities. Based on these facts, the current study aims to find out the total viable bacterial count and pathogenic load of various types of apples consumed in Bangladesh, drug resistance properties of the suspected pathogens, and finally to determine the antibacterial activities of these apple samples.

Prevalence of pathogenic microorganisms. Hazard Analysis and Critical Control Point-Total Quality Management (HACCP-TQM) technical guidelines rates microbial quality for raw foods containing aerobic plate count of $<10^{4} \mathrm{cfu} / \mathrm{g}$ as "Good", $10^{4}-5 \times 10^{6}$ $\mathrm{cfu} / \mathrm{g}$ as "Average", $5 \times 10^{6}-5 \times 10^{7} \mathrm{cfu} / \mathrm{g}$ as "Poor" and $>5 \times 10^{7} \mathrm{cfu} / \mathrm{g}$ as "Spoilt". Several previous studies showed that Escherichia coli, Salmonella spp., Shigella spp., Staphylococcus aureus, Vibrio spp. and fungi were frequent in a variety of fruits and vegetables samples (14, 22). However, in this study, after enrichment Vibrio spp. was estimated within a range of $3.5 \times 10^{3}-3.2 \times 10^{6} \mathrm{cfu} / \mathrm{g}$ while Salmonella and Shigella spp. were found in between $2 \times 10^{4}-5.6 \times 10^{6} \mathrm{cfu} / \mathrm{g}$ and $1 \times 10^{4}-2.8 \times 10^{6} \mathrm{cfu} / \mathrm{g}$, respectively (Table 1). Presence E. coli, Salmonella spp. and Shigella spp. which are often in connection with poor sanitary practices and they put a pointer to a potential risk of food borne illness to consumers (5). Surprisingly total viable bacterial load (TVBC) in all the samples observed in this study was almost same, near about $10^{6} \mathrm{cfu} / \mathrm{g}$ whereas coliform, Pseudomonas and fungal count, all were within the range of $10^{6} \mathrm{cfu} / \mathrm{g}$. Coliforms are indicator organisms and counts of $10^{4}-10^{6} \mathrm{cfu} / \mathrm{g}$ reported in this work are a cause for concern, since the fruits are usually consumed without further processing. Staphylococcus spp. was present within the range of $1 \times 10^{4}-3.2 \times 10^{6} \mathrm{cfu} / \mathrm{g}$. Staphylococcus aureus is common food contaminants from man and the environment, their presence in food however, need to be controlled because they have been reported as cause of major food borne illnesses (4). The presence of these pathogenic organisms revealed the possibility of spreading enteric diseases to the consumers. Fungus presence indicates the presence of various mycotoxins in the fruit samples that may pose severe threat to the human health. A study performed by Maria et al., (28) showed variable fungal growth in between $3.8 \times 10^{4}-5.9 \times 10^{4} \mathrm{cfu} / \mathrm{g}$. Overall, fungal growth of the apple samples in this study was $10^{6} \mathrm{cfu} / \mathrm{g}$ in average (Table 1). The fungal isolates of apple fruits in another study Aspergillus spp; Penicillium spp, Rhizopus, Mucor spp are common environmental contaminants.

So, this study demonstrated the presence of different types of microorganisms with a high load in the apple samples. There are so many possibilities by which pathogens may come in contact with the fruits such as the crop land, organic fertilizers, irrigating water, packaging materials, transport vehicles etc. Besides, unhygienic personnel practicing and processing of the fruits and their storage in such a condition which favors microbial growth might also be responsible for such spoilage of fruits. The contaminating pathogens are the reason for various types of enteric diseases as well as serious intoxications in human health.

Antibiotic susceptibility patterns of pathogens found in apple samples. Drug resistance is a serious problem in these days that is becoming more and more threat for the global public health. The antibiogram study showed, although some of the isolates were susceptible towards some antibiotics, several other antibiotics were proved ineffective, indicating the risk of the emerging resistant isolates causing health hazards. Most of the isolates were 
TABLE 1. Isolation and enumeration of microorganisms in the local market apple samples

\begin{tabular}{|c|c|c|c|c|c|c|c|c|c|}
\hline $\begin{array}{l}\text { Apple } \\
\text { sample }\end{array}$ & $\begin{array}{c}\text { Total } \\
\text { viable } \\
\text { bacteria } \\
\text { (cfu/g) }\end{array}$ & $\begin{array}{l}\text { Fungi } \\
\text { (cfu/g) }\end{array}$ & $\begin{array}{c}\text { Escherichia. } \\
\text { coli } \\
\text { (cfu/g) }\end{array}$ & $\begin{array}{c}\text { Klebsiella } \\
\text { spp. } \\
\text { (cfu/g) }\end{array}$ & $\begin{array}{c}\text { Salmonella } \\
\text { spp. } \\
\text { (cfu/g) }\end{array}$ & $\begin{array}{c}\text { Shigella } \\
\text { spp. } \\
\text { (cfu/g) }\end{array}$ & $\begin{array}{l}\text { Staphylococcus } \\
\text { spp (cfu/g) }\end{array}$ & $\begin{array}{l}\text { Pseudomonas } \\
\operatorname{spp}(\mathrm{cfu} / \mathrm{g})\end{array}$ & $\begin{array}{l}\text { Vibrio } \\
\text { spp } \\
(\mathbf{c f u} / \mathrm{g})\end{array}$ \\
\hline $\begin{array}{l}\text { Golden } \\
\text { delicious } 1\end{array}$ & $3.8 \times 10^{5}$ & $2.2 \times 10^{5}$ & $6.0 \times 10^{5}$ & 0 & $2.8 \times 10^{6}$ & $1.6 \times 10^{4}$ & $1.08 \times 10^{6}$ & $1 \times 10^{5}$ & $9.2 \times 10^{5}$ \\
\hline $\begin{array}{l}\text { Golden } \\
\text { delicious } 2\end{array}$ & $5.2 \times 10^{6}$ & $9 \times 10^{5}$ & $1.5 \times 10^{5}$ & $1.0 \times 10^{5}$ & $2.0 \times 10^{4}$ & $2.0 \times 10^{5}$ & $2.8 \times 10^{5}$ & $5 \times 10^{5}$ & $1 \times 10^{4}$ \\
\hline $\begin{array}{l}\text { Red } \\
\text { Delicious } 1\end{array}$ & $4.4 \times 10^{5}$ & $3.6 \times 10^{6}$ & $4.2 \times 10^{5}$ & 0 & $3.8 \times 10^{5}$ & $2.8 \times 10^{6}$ & $5.4 \times 10^{5}$ & $6.0 \times 10^{6}$ & $5.2 \times 10^{5}$ \\
\hline $\begin{array}{l}\text { Red } \\
\text { Delicious } 2\end{array}$ & $3.5 \times 10^{5}$ & $4.5 \times 10^{5}$ & $2 \times 10^{5}$ & 0 & 0 & $1 \times 10^{4}$ & $2.3 \times 10^{5}$ & $8 \times 10^{5}$ & $3.2 \times 10^{6}$ \\
\hline $\begin{array}{l}\text { Honey } \\
\text { Crisp } 1\end{array}$ & $1.6 \times 10^{6}$ & $8.4 \times 10^{5}$ & $4.5 \times 10^{5}$ & $2.2 \times 10^{5}$ & 0 & $3 \times 10^{4}$ & $1.3 \times 10^{6}$ & $1.2 \times 10^{6}$ & $1.4 \times 10^{5}$ \\
\hline $\begin{array}{l}\text { Honey } \\
\text { Crisp } 2\end{array}$ & $2.2 \times 10^{6}$ & $3.12 \times 10^{6}$ & $7.2 \times 10^{5}$ & 0 & $6.2 \times 10^{5}$ & $2.8 \times 10^{6}$ & $1 \times 10^{4}$ & $3.2 \times 10^{6}$ & $2.8 \times 10^{6}$ \\
\hline Fuji & $4.7 \times 10^{6}$ & $4.4 \times 10^{6}$ & $1.6 \times 10^{6}$ & 0 & $4.9 \times 10^{6}$ & 0 & $3.2 \times 10^{6}$ & $6 \times 10^{4}$ & $4.2 \times 10^{6}$ \\
\hline Gala & $3.8 \times 10^{5}$ & $5.4 \times 10^{7}$ & $1.8 \times 10^{5}$ & 0 & $3.7 \times 10^{5}$ & 0 & $4.1 \times 10^{4}$ & $4.2 \times 10^{5}$ & $3.5 \times 10^{3}$ \\
\hline Braeburn & $3.1 \times 10^{6}$ & $3.1 \times 10^{6}$ & $8.2 \times 10^{5}$ & 0 & $5.6 \times 10^{6}$ & 0 & $2 \times 10^{4}$ & $4.2 \times 10^{6}$ & $8.6 \times 10^{5}$ \\
\hline Paula Red & $3.5 \times 10^{5}$ & $9.8 \times 10^{5}$ & $2.2 \times 10^{5}$ & $3.5 \times 10^{5}$ & 0 & $1.2 \times 10^{5}$ & $6 \times 10^{4}$ & $9 \times 10^{5}$ & $1 \times 10^{4}$ \\
\hline
\end{tabular}

TABLE 2. Antibiogram of the different bacterial isolates collected from apple samples.

\begin{tabular}{|c|c|c|c|c|c|c|c|c|}
\hline Antibiotic & E. coli & $\begin{array}{l}\text { Klebsiella } \\
\text { spp. }\end{array}$ & $\begin{array}{l}\text { Pseudomonas } \\
\text { spp. }\end{array}$ & $\begin{array}{l}\text { Salmonella } \\
\text { spp. }\end{array}$ & $\begin{array}{l}\text { Staphylococcus } \\
\text { spp. }\end{array}$ & Listeria spp. & $\begin{array}{l}\text { Vibrio } \\
\text { spp. }\end{array}$ & Bacillus spp. \\
\hline $\begin{array}{l}\text { Gentamicin }(\mathrm{CN}) \\
10 \mu \mathrm{g}\end{array}$ & $\begin{array}{l}20 \mathrm{~mm} \\
(\mathrm{~S})\end{array}$ & $\begin{array}{l}17 \mathrm{~mm} \\
(\mathrm{~S})\end{array}$ & $\begin{array}{l}20 \mathrm{~mm} \\
(\mathrm{~S})\end{array}$ & $\begin{array}{l}19 \mathrm{~mm} \\
(\mathrm{~S})\end{array}$ & $\begin{array}{l}14 \mathrm{~mm} \\
\text { (I) }\end{array}$ & $\begin{array}{l}17 \mathrm{~mm} \\
(\mathrm{~S})\end{array}$ & $\begin{array}{l}23 \mathrm{~mm} \\
(\mathrm{~S})\end{array}$ & $\begin{array}{l}17 \mathrm{~mm} \\
(\mathrm{~S})\end{array}$ \\
\hline $\begin{array}{l}\text { Ampicillin(AMP) } \\
10 \mu \mathrm{g}\end{array}$ & $\begin{array}{c}13 \mathrm{~mm} \\
(\mathrm{R})\end{array}$ & $\begin{array}{c}12 \mathrm{~mm} \\
(\mathrm{R})\end{array}$ & $\begin{array}{c}11 \mathrm{~mm} \\
(\mathrm{R})\end{array}$ & $\begin{array}{c}9 \mathrm{~mm} \\
(\mathrm{R})\end{array}$ & $\begin{array}{c}13 \mathrm{~mm} \\
(\mathrm{R})\end{array}$ & $\begin{array}{c}12 \mathrm{~mm} \\
(\mathrm{R})\end{array}$ & $\begin{array}{c}34 \mathrm{~mm} \\
(\mathrm{~S})\end{array}$ & $\begin{array}{c}8 \mathrm{~mm} \\
(\mathrm{R})\end{array}$ \\
\hline $\begin{array}{l}\text { Amoxicillin } \\
\text { (AML) } 10 \mu \mathrm{g}\end{array}$ & $\begin{array}{c}12 \mathrm{~mm} \\
(\mathrm{R})\end{array}$ & $\begin{array}{l}26 \mathrm{~mm} \\
(\mathrm{~S})\end{array}$ & $\begin{array}{c}13 \mathrm{~mm} \\
(\mathrm{R})\end{array}$ & $\begin{array}{l}15 \mathrm{~mm} \\
\text { (I) }\end{array}$ & $\underset{(\mathrm{R})}{12 \mathrm{~mm}}$ & $\underset{(\mathrm{R})}{11 \mathrm{~mm}}$ & $\begin{array}{c}16 \mathrm{~mm} \\
\text { (I) }\end{array}$ & $\begin{array}{l}9 \mathrm{~mm} \\
(\mathrm{R})\end{array}$ \\
\hline $\begin{array}{l}\text { Chloramphenicol } \\
\text { (C) } 30 \mu \mathrm{g}\end{array}$ & $\begin{array}{l}26 \mathrm{~mm} \\
(\mathrm{~S})\end{array}$ & $\begin{array}{l}27 \mathrm{~mm} \\
(\mathrm{~S})\end{array}$ & $\begin{array}{l}30 \mathrm{~mm} \\
(\mathrm{~S})\end{array}$ & $\begin{array}{l}25 \mathrm{~mm} \\
(\mathrm{~S})\end{array}$ & $\begin{array}{l}19 \mathrm{~mm} \\
(\mathrm{~S})\end{array}$ & $\begin{array}{l}20 \mathrm{~mm} \\
(\mathrm{~S})\end{array}$ & $\begin{array}{l}20 \mathrm{~mm} \\
(\mathrm{~S})\end{array}$ & $\begin{array}{l}23 \mathrm{~mm} \\
(\mathrm{~S})\end{array}$ \\
\hline $\begin{array}{l}\text { Cefixime (CFM) } \\
5 \mu \mathrm{g}\end{array}$ & $\begin{array}{c}12 \mathrm{~mm} \\
(\mathrm{R})\end{array}$ & $\begin{array}{c}16 \mathrm{~mm} \\
\text { (I) }\end{array}$ & $\begin{array}{c}13 \mathrm{~mm} \\
(\mathrm{R})\end{array}$ & $\begin{array}{c}16 \mathrm{~mm} \\
\text { (I) }\end{array}$ & $\begin{array}{l}14 \mathrm{~mm} \\
(\mathrm{R})\end{array}$ & $\begin{array}{c}15 \mathrm{~mm} \\
(\mathrm{R})\end{array}$ & $\begin{array}{l}17 \mathrm{~mm} \\
\text { (I) }\end{array}$ & $\begin{array}{c}12 \mathrm{~mm} \\
(\mathrm{R})\end{array}$ \\
\hline $\begin{array}{l}\text { Ciprofloxacin } \\
\text { (CIP) } 5 \mu \mathrm{g}\end{array}$ & $\begin{array}{l}21 \mathrm{~mm} \\
(\mathrm{~S})\end{array}$ & $\begin{array}{l}21 \mathrm{~mm} \\
(\mathrm{~S})\end{array}$ & $\begin{array}{l}28 \mathrm{~mm} \\
(\mathrm{~S})\end{array}$ & $\begin{array}{l}30 \mathrm{~mm} \\
(\mathrm{~S})\end{array}$ & $\begin{array}{l}27 \mathrm{~mm} \\
(\mathrm{~S})\end{array}$ & $\begin{array}{c}17 \mathrm{~mm} \\
\text { (I) }\end{array}$ & $\begin{array}{l}27 \mathrm{~mm} \\
(\mathrm{~S})\end{array}$ & $\begin{array}{l}20 \mathrm{~mm} \\
\text { (I) }\end{array}$ \\
\hline $\begin{array}{l}\text { Erythromycin } \\
\text { (E) } 15 \mu \mathrm{g}\end{array}$ & $\begin{array}{l}26 \mathrm{~mm} \\
(\mathrm{~S})\end{array}$ & $\begin{array}{c}11 \mathrm{~mm} \\
(\mathrm{R})\end{array}$ & $\begin{array}{l}20 \mathrm{~mm} \\
(\mathrm{~S})\end{array}$ & $\begin{array}{l}20 \mathrm{~mm} \\
(\mathrm{~S})\end{array}$ & $\begin{array}{c}13 \mathrm{~mm} \\
(\mathrm{I})\end{array}$ & $\begin{array}{l}18 \mathrm{~mm} \\
(\mathrm{~S})\end{array}$ & $\begin{array}{c}14 \mathrm{~mm} \\
\text { (I) }\end{array}$ & $\begin{array}{l}25 \mathrm{~mm} \\
(\mathrm{~S})\end{array}$ \\
\hline $\begin{array}{l}\text { Imipenem } \\
\text { (IPM)10 } \mu \mathrm{g}\end{array}$ & $\begin{array}{l}31 \mathrm{~mm} \\
(\mathrm{~S})\end{array}$ & $\begin{array}{l}21 \mathrm{~mm} \\
(\mathrm{I})\end{array}$ & $\begin{array}{l}24 \mathrm{~mm} \\
(\mathrm{~S})\end{array}$ & $\begin{array}{c}17 \mathrm{~mm} \\
(\mathrm{R})\end{array}$ & $\begin{array}{l}40 \mathrm{~mm} \\
(\mathrm{~S})\end{array}$ & $\begin{array}{c}15 \mathrm{~mm} \\
(\mathrm{R})\end{array}$ & $\begin{array}{c}17 \mathrm{~mm} \\
(\mathrm{R})\end{array}$ & $\begin{array}{l}28 \mathrm{~mm} \\
(\mathrm{~S})\end{array}$ \\
\hline $\begin{array}{l}\text { Nalidixic acid } \\
\text { (NA) } 30 \mu \mathrm{g}\end{array}$ & $\begin{array}{c}11 \mathrm{~mm} \\
(\mathrm{R})\end{array}$ & $\begin{array}{l}15 \mathrm{~mm} \\
\text { (I) }\end{array}$ & $\begin{array}{l}25 \mathrm{~mm} \\
(\mathrm{~S})\end{array}$ & $\begin{array}{l}27 \mathrm{~mm} \\
(\mathrm{~S})\end{array}$ & $\begin{array}{l}14 \mathrm{~mm} \\
\text { (I) }\end{array}$ & $\begin{array}{c}12 \mathrm{~mm} \\
(\mathrm{R})\end{array}$ & $\begin{array}{c}13 \mathrm{~mm} \\
(\mathrm{R})\end{array}$ & $\begin{array}{c}9 \mathrm{~mm} \\
(\mathrm{R})\end{array}$ \\
\hline $\begin{array}{l}\text { Ceftazidime } \\
\text { (CAZ) } 30 \mu \mathrm{g}\end{array}$ & $\begin{array}{l}18 \mathrm{~mm} \\
\text { (I) }\end{array}$ & $\begin{array}{c}17 \mathrm{~mm} \\
(\mathrm{R})\end{array}$ & $\begin{array}{c}15 \mathrm{~mm} \\
(\mathrm{R})\end{array}$ & $\begin{array}{l}17 \mathrm{~mm} \\
\text { (I) }\end{array}$ & $\begin{array}{c}12 \mathrm{~mm} \\
(\mathrm{R})\end{array}$ & $\begin{array}{l}14 \mathrm{~mm} \\
(\mathrm{R})\end{array}$ & $\begin{array}{c}19 \mathrm{~mm} \\
\text { (I) }\end{array}$ & $\begin{array}{l}18 \mathrm{~mm} \\
\text { (I) }\end{array}$ \\
\hline $\begin{array}{l}\text { Cefuroxime } \\
\text { (CXM) } 30 \mu \mathrm{g}\end{array}$ & $\begin{array}{l}15 \mathrm{~mm} \\
\text { (I) }\end{array}$ & $\begin{array}{c}16 \mathrm{~mm} \\
\text { (I) }\end{array}$ & $\begin{array}{c}13 \mathrm{~mm} \\
(\mathrm{R})\end{array}$ & $\begin{array}{c}14 \mathrm{~mm} \\
(\mathrm{R})\end{array}$ & $\begin{array}{c}13 \mathrm{~mm} \\
(\mathrm{R})\end{array}$ & $\begin{array}{c}12 \mathrm{~mm} \\
(\mathrm{R})\end{array}$ & $\begin{array}{c}17 \mathrm{~mm} \\
\text { (I) }\end{array}$ & $\begin{array}{l}9 \mathrm{~mm} \\
(\mathrm{R})\end{array}$ \\
\hline $\begin{array}{l}\text { Vancomycin } \\
\text { (VA) } 30 \mu \mathrm{g}\end{array}$ & $\begin{array}{c}11 \mathrm{~mm} \\
(\mathrm{R})\end{array}$ & $\begin{array}{c}9 \mathrm{~mm} \\
(\mathrm{R})\end{array}$ & $\begin{array}{l}11 \mathrm{~mm} \\
(\mathrm{R})\end{array}$ & $\begin{array}{l}14 \mathrm{~mm} \\
\text { (I) }\end{array}$ & $\begin{array}{c}8 \mathrm{~mm} \\
(\mathrm{R})\end{array}$ & $\begin{array}{c}12 \mathrm{~mm} \\
\text { (I) }\end{array}$ & $\begin{array}{c}9 \mathrm{~mm} \\
(\mathrm{R})\end{array}$ & $\begin{array}{c}13 \mathrm{~mm} \\
\text { (I) }\end{array}$ \\
\hline
\end{tabular}


TABLE 2. Antibiogram of the different bacterial isolates collected from apple samples (continued)

\begin{tabular}{|c|c|c|c|c|c|c|c|c|}
\hline Antibiotic & E. coli & $\begin{array}{l}\text { Klebsiella } \\
\text { spp. }\end{array}$ & $\begin{array}{l}\text { Pseudomonas } \\
\text { spp. }\end{array}$ & $\begin{array}{l}\text { Salmonella } \\
\text { spp. }\end{array}$ & $\begin{array}{l}\text { Staphylococcus } \\
\text { spp. }\end{array}$ & Listeria spp. & $\begin{array}{l}\text { Vibrio } \\
\text { spp. }\end{array}$ & Bacillus spp. \\
\hline $\begin{array}{l}\text { Penicillin (P) } 10 \\
\mu \mathrm{g}\end{array}$ & $\begin{array}{c}13 \mathrm{~mm} \\
(\mathrm{R})\end{array}$ & $\underset{(\mathrm{R})}{12 \mathrm{~mm}}$ & $\begin{array}{c}11 \mathrm{~mm} \\
(\mathrm{R})\end{array}$ & $\begin{array}{l}9 \mathrm{~mm} \\
(\mathrm{R})\end{array}$ & $\begin{array}{c}8 \mathrm{~mm} \\
(\mathrm{R})\end{array}$ & $\begin{array}{c}12 \mathrm{~mm} \\
(\mathrm{R})\end{array}$ & $\underset{(\mathrm{R})}{11 \mathrm{~mm}}$ & $\begin{array}{c}8 \mathrm{~mm} \\
(\mathrm{R})\end{array}$ \\
\hline $\begin{array}{l}\text { Oxacillin }(O X) 1 \\
\mu \mathrm{g}\end{array}$ & $\begin{array}{l}14 \mathrm{~mm} \\
(\mathrm{R})\end{array}$ & $\begin{array}{c}12 \mathrm{~mm} \\
(\mathrm{R})\end{array}$ & $\begin{array}{c}11 \mathrm{~mm} \\
(\mathrm{R})\end{array}$ & $\begin{array}{l}9 \mathrm{~mm} \\
(\mathrm{R})\end{array}$ & $\begin{array}{l}7 \mathrm{~mm} \\
(\mathrm{R})\end{array}$ & $\begin{array}{c}12 \mathrm{~mm} \\
(\mathrm{R})\end{array}$ & $\begin{array}{c}13 \mathrm{~mm} \\
(\mathrm{R})\end{array}$ & $\begin{array}{l}7 \mathrm{~mm} \\
(\mathrm{R})\end{array}$ \\
\hline $\begin{array}{l}\text { Tetracycline } \\
\text { (TE) } 30 \mu \mathrm{g}\end{array}$ & $\begin{array}{l}15 \mathrm{~mm} \\
(\mathrm{~S})\end{array}$ & $\begin{array}{l}15 \mathrm{~mm} \\
(\mathrm{~S})\end{array}$ & $\begin{array}{l}22 \mathrm{~mm} \\
(\mathrm{~S})\end{array}$ & $\begin{array}{l}25 \mathrm{~mm} \\
(\mathrm{~S})\end{array}$ & $\begin{array}{l}13 \mathrm{~mm} \\
\text { (I) }\end{array}$ & $\begin{array}{l}9 \mathrm{~mm} \\
(\mathrm{R})\end{array}$ & $\begin{array}{c}14 \mathrm{~mm} \\
\text { (I) }\end{array}$ & $\begin{array}{l}12 \mathrm{~mm} \\
\text { (I) }\end{array}$ \\
\hline $\begin{array}{l}\text { Azithromycin } \\
\text { (AZM) } 15 \boldsymbol{\mu g}\end{array}$ & $\begin{array}{l}17 \mathrm{~mm} \\
(\mathrm{~S})\end{array}$ & $\begin{array}{l}17 \mathrm{~mm} \\
(\mathrm{~S})\end{array}$ & $\begin{array}{c}11 \mathrm{~mm} \\
(\mathrm{R})\end{array}$ & $\begin{array}{l}15 \mathrm{~mm} \\
(\mathrm{~S})\end{array}$ & $\begin{array}{l}18 \mathrm{~mm} \\
(\mathrm{~S})\end{array}$ & $\begin{array}{l}9 \mathrm{~mm} \\
(\mathrm{R})\end{array}$ & $\begin{array}{c}16 \mathrm{~mm} \\
(\mathrm{~S})\end{array}$ & $\begin{array}{l}28 \mathrm{~mm} \\
(\mathrm{~S})\end{array}$ \\
\hline $\begin{array}{l}\text { Penicillin G (P } \\
\text { G) } 10 \mu \mathrm{g}\end{array}$ & $\begin{array}{l}15 \mathrm{~mm} \\
(\mathrm{R})\end{array}$ & $\begin{array}{c}14 \mathrm{~mm} \\
(\mathrm{R})\end{array}$ & $\begin{array}{c}13 \mathrm{~mm} \\
(\mathrm{R})\end{array}$ & $\begin{array}{c}14 \mathrm{~mm} \\
(\mathrm{R})\end{array}$ & $\begin{array}{c}13 \mathrm{~mm} \\
(\mathrm{R})\end{array}$ & $\begin{array}{c}12 \mathrm{~mm} \\
(\mathrm{R})\end{array}$ & $\begin{array}{c}11 \mathrm{~mm} \\
(\mathrm{R})\end{array}$ & $\begin{array}{l}9 \mathrm{~mm} \\
(\mathrm{R})\end{array}$ \\
\hline $\begin{array}{l}\text { Streptomycin } \\
\text { (S) } 10 \mu \mathrm{g}\end{array}$ & $\begin{array}{l}9 \mathrm{~mm} \\
(\mathrm{R})\end{array}$ & $\begin{array}{c}8 \mathrm{~mm} \\
(\mathrm{R})\end{array}$ & $\begin{array}{l}7 \mathrm{~mm} \\
(\mathrm{R})\end{array}$ & $\begin{array}{c}8 \mathrm{~mm} \\
(\mathrm{R})\end{array}$ & $\begin{array}{l}9 \mathrm{~mm} \\
(\mathrm{R})\end{array}$ & $\begin{array}{l}15 \mathrm{~mm} \\
(\mathrm{~S})\end{array}$ & $\begin{array}{l}9 \mathrm{~mm} \\
(\mathrm{R})\end{array}$ & $\begin{array}{l}14 \mathrm{~mm} \\
(\mathrm{~S})\end{array}$ \\
\hline $\begin{array}{l}\text { Ceftriaxone } \\
\text { (CRO) } 30 \mu \mathrm{g}\end{array}$ & $\begin{array}{l}19 \mathrm{~mm} \\
(\mathrm{R})\end{array}$ & $\begin{array}{c}18 \mathrm{~mm} \\
(\mathrm{R})\end{array}$ & $\begin{array}{c}17 \mathrm{~mm} \\
(\mathrm{R})\end{array}$ & $\begin{array}{c}16 \mathrm{~mm} \\
(\mathrm{R})\end{array}$ & $\begin{array}{l}15 \mathrm{~mm} \\
(\mathrm{R})\end{array}$ & $\begin{array}{l}14 \mathrm{~mm} \\
(\mathrm{R})\end{array}$ & $\begin{array}{l}20 \mathrm{~mm} \\
\text { (I) }\end{array}$ & $\begin{array}{l}18 \mathrm{~mm} \\
(\mathrm{R})\end{array}$ \\
\hline
\end{tabular}

$\mathrm{R}=$ Resistant $; \mathrm{S}=$ Sensitive; I= Intermediate

showed that Staphylococcus spp. were highly resistant against ampicillin, amoxicillin, penicillin. vancomycin, enoxairtfce, cefixime, oxacillin, ceftazidime, oximecefur and streptomycine. This result is quite similar with this study (Table 2). High resistance of Vibrio spp. against ampicillin, chloramphenicol, nalidixic aciderythromycin, ciprofloxacin, penicillin, streptomycin, vancomycin, cefixime and cefuroxime has been indicated by Acharjee et al. (8), whereas in this study variable susceptibility pattern were obtained against the different antibiotics for Salmonella and Vibrio isolates (Table 2). Pseudomonas spp and Klebsiella spp. were also found to be resistant against most of the antibiotics (Table 2).

Antibacterial activity of apple samples. Antimicrobial activity was determined by using the agar well diffusion methods as previously demonstrated (26) against eight common bacterial laboratory isolates (Bacillus spp., Pseudomonas spp., Vibrio spp., Escherichia coli, Klebsiella spp., Staphylococcus spp., Listeria spp. and Salmonella spp.). Previous study showed that, the apple skin portions were found to exhibit moderate anti-bacterial activity against Pseudomonas spp. and Listeria spp. with a relatively lower activity against Klebsiella spp.; while the flesh portions showed to moderately active against Pseudomonas spp. and Staphylococcus spp. and arelatively lower activity against Listeria spp. and Aeromonas spp. (29). In the current study no antibacterial activity was observed to be present in the experimented samples.

\section{CONCLUSION}

Overall, the findings of this study clearly indicate a complete bacteriological profile of local market apples, which is of public health significance. Further studies with some advanced molecular settings for the better detection of the pathogens as well as some good solvent extraction methods need to be established to use apple as a potential therapeutic agent which may reduce the use of conventional drugs for the remedy of various diseases.

\section{ACKNOWLEDGEMENT}

Authors are thankful to the Department of Microbiology, Stamford University Bangladesh for all the technical help and authors also acknowledge the research groups whose findings have been used to write this article.

\section{REFERENCES}

1. Fatema N, Acharjee M, Noor R. 2013. Microbiological profiling of imported apples and demonstration of bacterial survival capacity through in vitro challenge test. Ame. J. Microbiol. Res. 1 (4): 98-104.

2. Mukhtar A, Gilani AH, Bhatty N. 2010. Some nutritional and microbiological aspects of apples of common varieties available for household consumption. J. Anim. Plant Sci. 20 (4): 253-257.

3. Olaimat AN, Holley RA. 2002. Factors influencing the microbial safety of fresh produce: A review. Food Microbiol. 32: 1-19.

4. Mudgil S, Argawal D, Ganguli A. 2004. Microbiological analysis of street vended fresh squeezed carrot and kinnow mandarin juice in Patiala City. India. Internet J. Food Safety. 3: 1-3.

5. Aycicek H, Oguz U, Karci K. 2006. Determination of total aerobic and indicator bacteria on some raw eaten vegetables from whole sellers in Ankara. Turkey. Int. J. Hyg. Environ. Health. 209: 197-201.

6. Mathew AG, Cissell R, Liamthong S. 2007. Antibiotic resistance in bacteria associated with food animals: a United States perspective of livestock 
production. Foodborne Path. Dis. 4 (2): 115-133.

7. Allerberger F, Mittermayer H. 2008. Antimicrobial stewardship. Clin. Microbiol. Infect. Doi:10.1111/j.1469-0691.2007.01929.x

8. Acharjee M, Jahan F, Rahman F, Noor R. 2013. Bacterial prolifiration in municipal water supplied in Mirpur locality of Dhaka city, Bangladesh. CLEAN- Soil Air Water. 41: 1-8.

9. Dutta S, Hassan MR, Rahman F, Jilani MSA, Noor R. 2013. Study of antimicrobial susceptibility of clinically significant microorganisms isolated from selected areas of Dhaka, Bangladesh. Bang. J. Med. Sci. 12 (1): 34-42.

10. Noor R, Acharjee M, Ahmed T, Das KK, Paul L, Munshi SK, et al. 2013. Microbiological study of major sea fish available in local markets of Dhaka city, Bangladesh. J. Microbiol. Biotechnol. Food Sci. 2 (4): 24202430.

11. Khan SA, Feroz F, Noor R. 2013. Study of extended spectrum $\beta$-lactamase producing bacteria from urinary tract infection in Dhaka city, Bangladesh. Tzu Chi Med. J. 25 (1): 39-42.

12. Bonjar S. 2004. Evaluation of antibacterial properties of some medicinal plants used in Iran. Journal of Ethnopharmacology. 94 (2-3): 301-5

13. Mwambete KD, Juistin TM, Faleabbas SF. 2009. Microbiological assessment of commercially available quinine syrups and water for inections in Dares Salaamm Tanzania. Tropical Journal of pharmaceutical Research. 8 (5): 441-447.

14. Ahmed T, Baidya S, Sharma BC, Malek MM, Das KK, Acharjee M. 2013. Identification of drug-resistant bacteria among export quality shrimp samples in Bangladesh. Asian Journal of Microbiology, Biotechnology and Environmental Science. 15 (4): 31-36.

15. Shan B, Cai Y, Brooks JD, Corke H. 2007. The in vitro antibacterial activity of dietary spice and medicinal herb extracts. International Journal of Food Microbiology. 117: 112

16. Haslam E. 1996. Natural polyphenols (vegetable tannins) as drugs: possible modes of action. J. Nat. Prod. 59 (2): 205-15.

17. Li Y, Guo C, Yang J, Wei J, Xu J, Cheng S. 2006. Evaluation of antioxidant properties of pomegranate peel extract in comparison with pomegranate pulp extract. Food Chemistry. 96: 254-260.
18. American Public Health Association (APHA). 1998. Standard methods fo the examination of water and wastewater. American Public Health Association, Washington, D.C.

19. Cappuccino JG, Sherman N. 1996. Microbiology - A laboratory manual. The Benjamin/Cummings Publishing Co., Inc., Menlo Park, California.

20. Polcovnicu C, Ionescu L, Bahrim G. 2008. Confirmation and identification of Listeria species from fresh lettuce. Romanian Biotechnol. Let. 13 (6): 32 36.

21. Colwell RR. 2000. Non-culturable microorganisms in the environment. American Society of Microbiology, Washington DC, USA.

22. Rahman F, Noor R. 2012. Prevalence of pathogenic bacteria in common salad vegetables of Dhaka metropolis. Ban. J. Bot. 41 (2): 150-162.

23. Alfrad EB. 2007. Bensons microbiological applications. Mcgraw-Hill Book Company, New York.

24. Bauer AW, Kirby WMM, Sherris JC, Tierch M. 1968. Antibiotic susceptibility testing by a standardized single disc method. American J. Clin. Pathol. 45 (4): 493-496.

25. Munshi SK, Rahman MM, Noor R. 2012. Detection of virulence potential of diarrheagenic Escherichia coli isolated from surface water of rivers surrounding Dhaka city. J. Bang. Acad. Sci. 36 (2): 109-121.

26. Jagessar RC, Mars A, Gones G. 2008. Selective antimicrobial properties of leaf extract against various microorganisms using disc diffusion and agar well diffusion method. J. Nat. Sci. 6 (2): 24-38.

27. Hussain A, Wahab S, Zarin I, Hussain MDS. 2010. Antibacterial activity of the leaves of Cocciniaindica (W. and A) of India. Adv. Biol. Res. 4 (5): 241 248

28. Afroz H, Ahmed T, Uddin MA. 2015. Microbiological analysis and antibacterial activity of pear samples. Stamford Journal of Microbiology. 5 (1): $1-4$.

29. Tahera J, Feroz F, Senjuti JD, Das KK, Noor R. 2014. Assessment of microbiological contamination and the in vitro demonstration of the antibacterial traits of the commonly available local fruit blend within Dhaka Metropolis. Journal of Pharmacognosy and Phytochemistry. 3(1): 73-77. 